\title{
Light Management in Advanced Photoelectrodes for Efficient Charge Collection and Light Absorption
}

\author{
Shihe Yang ${ }^{1,2}$ \\ ${ }^{1}$ Guangdong Key Lab of Nano-Micro Material Research, School of Chemical Biology and Biotechnology \\ Shenzhen Graduate School, Peking University, Shenzhen, China. \\ ${ }^{2}$ Department of Chemistry, The Hong Kong University of Science and Technology \\ Clear Water Bay, Kowloon, Hong Kong, China \\ chsyang@pku.edu.cn
}

\section{Extended Abstract}

Photoelectrochemical (PEC) water splitting is a promising technique to directly convert solar energy into hydrogen energy for storage and transportation. PEC devices are designed to efficiently collect the photogenerated, energy- and spaceseparated charges. The semiconductor-electrolyte interface plays an important role in PEC, at which charge carriers drive various chemical reactions, such as water splitting and $\mathrm{CO}_{2}$ reduction. Unfortunately, current PEC cells could hardly reach an open circuit photovoltage of $1.23 \mathrm{~V}$ for water splitting. To fill this gap, tandem devices with a PEC electrode connecting with another photoelectrode or a PV cell is brought to the table as a potential solution.

Achieving a high photocurrent is closely related to light absorption and charge collection. With a sufficient light absorption and full charge collection, this current should reach its optical limit. Unfortunately, it has been difficult to achieve simultaneously sufficient light absorption and efficient charge collection, i.e., increasing the thickness of a flat absorber to optimize light absorption only erode its ability to collect the photo-generated charges due to the long travel distance to charge collector. As a result, state-of-the-art current density reported to date is still far away from the limit. Studies have pointed out the charge conduction in most of metal oxide for PEC water splitting involve small polaron hopping mechanism with characteristically low mobility. This inevitably results in a very thin optimal thickness to collect charge. To overcome this dilemma, photoelectrodes with nanostructures have been employed. Researchers also attempted to incorporate wide-bandgap charge collecting scaffolds with very thin narrow band gap surface coating to build a hierarchical structure in hope of achieving both adequate light absorption and good charge collection. However, the goal of these strategies is mainly focused on the modification of electrical properties. Besides the charge transport process, light propagation and interaction with the absorber is also very important. Light trapping is intended to use special structures of the absorber to multiply the light pathways with two major desirable effects: 1) increasing the overall absorption in a thin film, and 2) modifying the light intensity redistribution to fit with the charge transport characteristic of a given photoelectrode for optimal charge collection. Therefore, the rationales for designing a light trapping structure will be: 1) with more light absorbed, more carriers will be generated; 2) by rationally focusing more light to where free charges can be efficiently collected, more carriers will be free of recombination.

In this presentation, I will introduce strategies to cope with the absorption-collection dilemma from a light management perspective, with a particular focus on a simultaneous achievement of efficient light harvesting and charge collection. I will discuss photonic structures for light trapping and modulation in PEC water splitting. To highlight the importance of understanding charge collection characteristic to light trapping design, I will present case studies on charge collection properties of some absorber materials and the corresponding light trapping design.

\section{Acknowledgement}

The work is supported by the Guangdong Science and Technology Program (2017B030314002) and the Shenzhen Peacock Plan (KQTD2016053015544057). 


\section{References}

[1] S. Xiao, S. Yang, H. Chen, Z. Yang, X. Long, Z. Wang, Z. Zhu, Y. Qu, "Origin of the Different PEC Performance of Mesoporous $\mathrm{BiVO}_{4}$ Photoanodes between the $\mathrm{BiVO}_{4}$ and the FTO Side Illumination," J. Phys. Chem. C, vol. 119, no. 41, pp. 23350-23357, 2015.

[2] Y. Qiu, S. Yang, S.-F. Leung, Q. Zhang, B. Hua, Q. Lin, Z. Wei, K.-H. Tsui, Y. Zhang, Z. Fan, "Efficient Photoelectrochemical Water Splitting with Ultrathin Films of Hematite on Three-Dimensional Nanophotonic Structures," Nano Lett., vol. 14, no. 4, pp. 2123-2129, 2014. 